drug companies to pay-have all been looked at and variously used.

Linking the information in the record to various computer systems is a problem because there is not a single standard computer system in all districts. Luckily most districts use the National Child Health Computer System, but others do use alternatives. The design of certain of the pages concerned with the routine checks on the child and the immunisations done have been made similar to the 'screens' on the national computer system, with non-carbonated copies of these pages being sent centrally for the information to be put onto the district health authority computer system. Problems arise however as this compromises the records' 'user friendliness' for parents.

There is the question as to whether there is a need to keep parallel records. General practitioners are legally bound to keep there own standard practice records on their own patients. What records other professionals have to keep (health visitors, community doctors, therapists, etc) depends on the needs of individual practitioners in their care of the individual children. Where there is a question of possible or actual child abuse it is recommended that parellel records should automatically be kept, but that the parents should be informed of this.

\section{What of the future?}

With recent recommendations from the Department of Health and the Foundation for the Study of Infant Deaths consideration will now be given to having a page/pages devoted to the prevention of sudden infant death syndrome, including information concerning the position a child is put down to sleep and the hazards of smoking. Sometime in the future, after further research, consideration may also be given to having a modified system of aiding parents to recognise significant signs and symptoms of illness in their children.

Pages for the record concerning the health and development of school age children are under consideration. There are, however, a number of problems. First is the whole question of the future provision of school health services. Second is the fact that sometime during the school age years, the focus of responsibility for health shifts from the parents to the child. Third is the lack of available research showing the value of detailed health promotion in this age group. All this also raises the issue as to when the record should be given to a young adult, and for the more distant future, whether the record could continue to be used on into adult life.

A further proposition for the future is to keep a national 'library' of different pages for the record as developed by different people in different districts. The need for such a library containing all the variations on the national record has become obvious, but who should keep it and how it should be financed has yet to be settled.

There is also the need for further research to look at the costs, benefits, and drawbacks to further developments in the record. For instance new national centile charts are being drawn up in the light of the evidence that the present Tanner and Whitehouse charts are outdated. These new charts will be completely redesigned using information available from the various present databases concerned with the growth of children.

In conclusion, the development of a national personal child health record far from being a 'one off' concept is an ongoing dynamic entity that consumes much time and work, but also offers great rewards as long as it is recognised that the ideal should not stand in the way of putting into practice what is merely a great deal better than what was there before. How well it works does not only depend on its use by parents but even more so on its use by, and the value attached to it by, doctors and nurses both in the community and hospital, If it is valued by them, then the parents will value it too, will not loose it, and will bring it with them to all contacts with the primary and secondary care providers to share the information so as to ensure the better care of their child.

Community Health Offices,

AIDAN MACFARLANE

\section{Radcliffe Infirmary} Oxford $O X 26 H F$

1 Mayall B. Child Health Care. Living with children, working for children. London: Heinemann Nursing, 1989.

2 Spencer NJ. Parents recognition of the ill child. In: Macfarlane A, ed. Progress in child health. Vol 1. Edinburgh: Churchill Livingstone, 1984. 3 Mayall B. Keeping children healthy. London: Allen and Unwin, 1986.

4 Sefi S, Macfarlane A. Child health clinics: why mothers attend. Health Visito 1985;58:129-30.

5 Saffin K, Macfarlane A. How well are parent held records kept and completed. Br 7 Gen Pract 1991;41:249-51.

6 Miller SA StJ. A trial of parent held child health records in the armed forces. BMF 1990;300:1046

7 British Paediatric Association. Report of the joint working party on professional and parent held records used in child health surveillance. London: British Paediatric Association, 1990.

\section{Medulloblastoma}

The treatment of childhood brain tumour is a subject that represents a major challenge and yet remains a major frustration to the paediatric oncologist. Medulloblastoma is a rare, highly malignant tumour usually arising in the vermis of the cerebellum. It accounts for approximately $30 \%$ of childhood brain tumours, or one child per 150000 per year ( 80 cases year in the UK). The tumour can occur at any age through childhood with a peak occurring around the age of 5 years. Males are slightly more often affected than females. Medulloblastoma is therefore a rare diagnosis in the majority of paediatric units and is a tumour of sufficient rarity that international cooperation has been essential to study possible treatment advances.

The histological origin of the medulloblastoma is uncertain. It may arise from embryonal rest cells left behind after the development and subsequent disappearance of the outer granular layer of the cerebellum in the roof of the fourth ventricle. An alternative theory is that medulloblastoma originates from undifferentiated cells in the subependymal region in the roof of the fourth ventricle. These cells have the ability to differentiate into neuronal and glial cells and are found in all ages and in various areas of the brain, in particular in the area of the fourth ventricle, in the cerebellum, and in those parts of the pallidum, nucleus caudatum, and thalamus that are next to the ventricles. Medulloblastoma-like tumours arise in these areas and this observation has led Rorke to suggest that the term 'primitive neuroectodermal tumour' (PNET) should be applied to all of these tumours. ${ }^{1}$ Other pathologists however disagree with this view and suggest that the term medulloblastoma should 
be reserved for tumours arising in the classical site, that is the cerebellum, and the term PNET be used for similar tumours arising elsewhere in the central nervous system.

Diagnosis is usually made by computed tomography after clinical suspicion of raised intracranial pressure. There is however no 'classical' appearance of medulloblastoma on a computed tomogram and the diagnosis must be histologically confirmed. ${ }^{2}$ The relief of raised intracranial pressure is a surgical emergency and is best achieved by removal of the tumour rather than by ventriculoperitoneal shunting, although this may at times be necessary. Once raised intracranial pressure is relieved it is essential to obtain imaging of the spine either by a computed tomographic myelogram or by a gadolinium enhanced magnetic resonance image. As many as $20 \%$ of childhood medulloblastomas have clinically unsuspected spinal deposits present at diagnosis. ${ }^{3}$ If present these require treatment with enhanced spinal radiotherapy doses and are an indicator of poor ultimate outcome.

Surgery alone is a never curative treatment for medulloblastoma and postoperative radiotherapy is essential. The tumour originates in contact with the cerebrospinal fluid and this is the pathway of metastatic spread. Radiotherapy therefore is needed to treat the tumour bearing area and also to treat 'prophylactically' the rest of the cranium and spine. Disease free survival is related to the dose of radiotherapy. The minimum dose to the tumour bearing area should be 55 Gy and the craniospinal dose $35 \mathrm{~Gy}$. Unfortunately these doses when delivered to the developing brain will often be accompanied by long term sequelae. Growth failure is common and is due to a combination of failure of spinal growth and growth hormone deficiency. Many studies have shown that there is loss of intellectual function which becomes more severe the younger the child is at the time of treatment. ${ }^{4}$

At the present time $60 \%$ of children with medulloblastoma can expect to be long term survivors when treated with maximal surgical removal of their tumour followed by radiotherapy. Current clinical research is therefore concentrated on two questions: (1) can this figure be improved upon and (2) can treatments with fewer long term sequelae be devised?

Chemotherapy has and is being studied in the context of international randomised clinical trials. Many chemotherapeutic agents have been shown to be effective in producing remissions in children with either recurrent medulloblastoma or in children with measurable residual disease after surgery and radiotherapy. The first randomised studies carried out in Europe and the USA showed that there appeared to be a marginal benefit when chemotherapy with vincristine and lomustine (CCNU, Lundbeck) was given after radiotherapy. ${ }^{5}$ The second generation of studies sought to exploit the time period between surgery and radiotherapy, which is a theoretically advantageous window of time in which to give chemotherapy. This has failed to show any advantage using the drugs and doses prescribed in the second European trial. ${ }^{7}$ Results of the similar American trial are awaited. The European and American studies sought to improve quality of survival by reducing the prophylactic craniospinal radiotherapy for children who appeared to have had complete surgical removal of the primary tumour and no demonstrable metastatic disease. Both studies have shown that this had a deleterious effect on survival and this approach has therefore been abandoned.

New studies about to be launched in Europe have been developed by the UK Children's Cancer Study Group and the International Society for Paediatric Oncology. For children over the age of 3 years intensive chemotherapy using carboplatin, cyclophosphamide, etoposide, and vincristine given after surgery and before radiotherapy will be compared with classical radiotherapy alone. For children under 3 years an intensive chemotherapeutic schedule lasting one year will be studied. Radiotherapy will be withheld during this time thus allowing time for the completion of development of the central nervous system. Once the child is beyond the third birthday, radiotherapy will be given to the tumour bearing area only, thus hopefully improving survival and quality of survival.

Regional Paediatric Oncology Unit,

CLIFFORD C BAILEY

Children's Day Hospital,

St fames's University Hospital,

Leeds LS9 7TF

1 Rorke LB. The cerebellar medulloblastoma and its relationship to primitive neuroectodermal tumours. F Neuropath Exp Neurol 1983;42:1-15.

2 Nelson M, Diebler C, St Claire-Forbes W. Paediatric medulloblastom atypical CT features at presentation in the SIOP II trial. Neuroradiolo 1991;33:140-2.

3 Deutsch M, Reigel DH. The value of myelography in the management of childhood medulloblastoma. Cancer 1980;45:2194-7.

4 Hoppe-Hirsch E, Renier D. Lellough-Tubiana A, Sainte-Rose C, PierreKhan A, Hirsch JF. Medulloblastoma in childhood: progressive intellectual Khan A, Hirsch JF. Medulloblastoma in child

5 Tait DM, Thornton-Jones H, Bloom HJ, Lemerle J, Morris-Jones P. Adjuvent chemotherapy for medulloblastoma: the first multicentre controlled trial of the International Society for Paediatric Oncology (SIOP 1). Eur $\mathcal{F}$ Cancer 1990;26:464-9.

6 Evans AE, Jenkin RD, Sposto R, et al. The treatment of medulloblastoma. Results of a prospective randomised trial of radiation therapy with and without CCNU, vincristine and prednisolone. $\mathcal{F}$ Neurosurg 1990;72:572-82

7 Gnekow AK, Bailey CC, Michaelis J, Wellek S, Kleihues O. SIOP/GPO medulloblastoma trial II med 84 annual status report. Med Pediatr Oncol 1991;19:435(abst) 